\title{
Design and Application of the Grab Operation Test System
}

\author{
Hao Zhou ${ }^{1, a^{*}}$, Xinming Zhou ${ }^{2, b}$ \\ ${ }^{1,2}$ School of Automation, Wuhan University of Technology, Wuhan 430070, P.R. China \\ a,b zhouhao901213@163.com
}

Keywords: gantry crane, grab, test system, DAQ card, working performance.

Abstract. This paper introduces a grab operation test system of a gantry crane and apply this system to compare the working performance of the new hollowed grab bucket with the traditional grab bucket. By using the data acquisition card (DAQ card), the test system collects the data of torque limiter and current of the motor. It implements real-time data display and storage with upper computer and database technology. Practice proves that this test system can truly reflect the grab operation rules, operational efficiency and stability.

\section{Introduction}

With the implementation and development of economic globalization, trade between different regions is increasingly frequent. It led to a large rise of the working load in port. One of the most important factors affecting the port handling capacity is the efficiency of the grab operation [1]. In order to further increase the efficiency of the grab operation, some colleges and research institutes tried to improve the grab control strategy or update the grab bucket [2]. Under this circumstance, a set of complete grab operation test system is needed to test and evaluate the performance of the grab operation.

\section{Principle of the Grab Operation Test System}

The control system of the grab uses PLC as the main controller to control four motors. Lifting motor is responsible for the rise and fall of the grab, open-close motor is responsible for the open and close of the grab, rotating motor charges of transferring material from the site to the destination while luffing motor adjusting the grab working range. The grab operation test system collects the data of torque limiter and the real-time current of the lifting motor and the open-close motor.

For the entire grabbing operation, it is required to be more cautious when operator closing the grab bucket. Overloading and other unstable situation are usually occurred at that time. So, the most important analysis of grab handling stability lies in the grab bucket closing process. At that time, the lifting motor and the open-close motor are working while the other two are not. For this reason, the grab operation test system should collect the data of torque limiter and the real-time current of lifting motor and open-close motor.

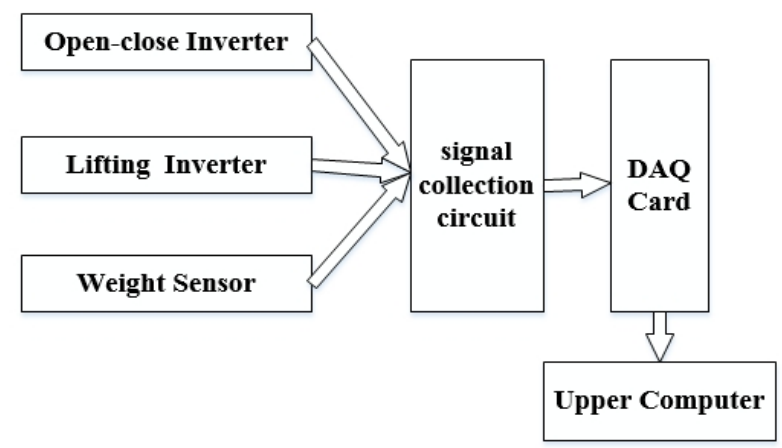

Fig.1. Diagram of the Grab Operation Test System

The test system is independent with the control system. By modifying the man-machine interface of the inverter, the AM port in inverter can be set as the standard $0 \sim 10 \mathrm{~V}$ voltage signal and corresponds 
to the motor current. Besides, the signal of the weight sensor is directly connected to the torque limiter, so the real-time weight is read from the torque limiter. The output data of the torque limiter is standard voltage signal and $10 \mathrm{~V}$ is corresponds to the rated lifting capacity of 30 tons.

The test system is illustrated in Fig.1.By utilizing the USB4711-A data acquisition card, the test system collects these data during the whole working period. In addition, it achieves the purpose of real-time display and storage of the data with the help of upper computer and database technology.

\section{Hardware Design of the System}

Introduction of the DAQ card. Data Acquisition (DAQ) is the use of computers to measure various physical quantities, such as: power, temperature, current and so on. With the rapid development of computer and digital electronic technology, data collection has become a key technology and is commonly used in many areas. Data acquisition card (DAQ card), a kind of computer expansion card, which has a function of data acquisition.

In the real practice, the data collected is standard $0-10 \mathrm{~V}$ voltage signal for 3 channels. Therefore, the system chooses the Advantech USB-4711A as the data acquisition card. It supports 12-bit resolution, 16 digital I / O channels and the sampling frequency is up to $100 \mathrm{ks} / \mathrm{s}$. What is more, each analog input can be selected within the range of $\pm 10 \mathrm{~V}$

Design of signal collection circuit. In the data acquisition process, there is a lot of external interference, and the voltage amplitude of the collected signal may be too big or too small, resulting in data distortion, so the output from the inverter should firstly pass a signal acquisition circuit. By isolating and filtering the noise wave, the circuit can ensure the safety of the data acquisition card effectively.

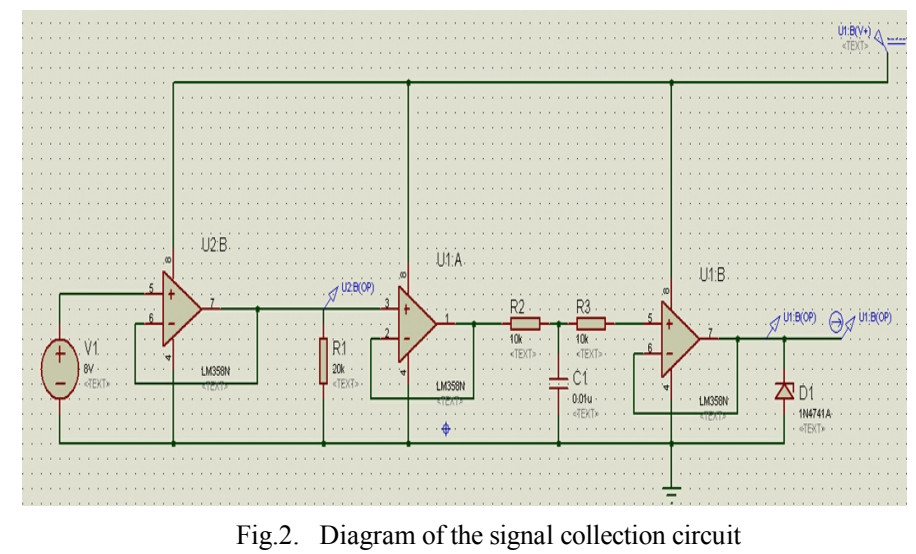

The signal acquisition circuit is as shown in Fig.2. At the input stage side, there is a voltage follower used to isolate the input terminal from the subsequent acquisition circuit, forming a protective effect on the circuit. Then, use a resistor of $20 \mathrm{Ohm}$ to get the impedance matching. Thirdly, insulate the filter circuit from the output stage with two operational amplifiers. Finally, adopt a resistor string diode voltage regulator circuit to maintain the analog signal output within the measuring range.

\section{Software Design of the System}

Based on the VB technology, the test system edit the PC interface for the real-time display of the collecting data. The data will also be stored in SQL Server and be exported in EXCEL format at last.

The built-in timer from VB can be used to set an appropriate sampling period. Implementation procedure is as follows:

Private Sub cmdRead_Click( )

flag $=0$

Timer1.Enabled $=$ True

End Sub

Private Sub Timer1_Timer() 


\section{Call cmdReadData}

\section{End Sub}

At the beginning of the data reading, the flag bit is set to zero and Timer1 starts. The timer only supports Timer event. Only when reaching the time fit for the demand of the Interval will trigger Timer event. Significantly, the function of the Timer event is to call cmdReadData to read and store the collecting data. In the program, the Interval value is set to 10 , that is to say, the collecting order will trigger every $10 \mathrm{~ms}$.

The actual sampling period is about $80 \mathrm{~ms}$ including the triggering time as well as the program execution time. In other words, the system collects 12-13 sets of data per second. By modifying the man-machine interface of the inverter, the AM port can be set as the standard $0 \sim 10 \mathrm{~V}$ voltage signal and corresponds to the motor current. However, in order to avoid over-current condition, the gain of the current analog output was adjusted to $80 \%$. That is to say, the $0-8 \mathrm{~V}$ voltage value is corresponds to the motor current value from zero to rated. Eq. 1 shows the mathematical relations.

$$
I_{a}=\frac{U_{c} I_{e}}{U_{0}}
$$

In this formula, $I_{a}$ stands for the real-time current of the motor; $U_{c}$ stands for the collected voltage signal ; $I_{e}$ stands for the rated current of the moto; ${ }^{U_{0}}$ stands for $8 \mathrm{~V}$, the voltage base value.

For example, if the collected voltage is $2 \mathrm{~V}$, the working current of the motor is 0.25 times the rated current.

\section{Application}

With the rapid development of domestic steel industry, imports of rare minerals, particularly nickel ore continue to grow. Nickel ore has two distinct features: First, the relative density is very small, usually in 0.9-1.5t / m3; the second is its high viscosity which causes strong adhesion easily [3].

At present, there are two main methods dealing with nickel ore:

(1)Traditional six-petaloid grab bucket, but with low loading efficiency.

(2)Excavator bucket, cooperate with the bauxite bucket, with higher loading efficiency but cost too much.

A research team has developed a new hollowed grab bucket to solve this problem, which is shown in Fig. 3. The grab operation test system can illustrate the difference between the new hollowed grab bucket with the traditional grab bucket and provide theoretical support for further improve of the grab bucket.

A grab experiment took place in China Tianjin port. We choose the 10t hollowed grab bucket and the $10 \mathrm{t}$ traditional grab bucket to do this experiment. In order to accurately reflect the differences between the two kinds of buckets, each bucket works for 20 cycles.

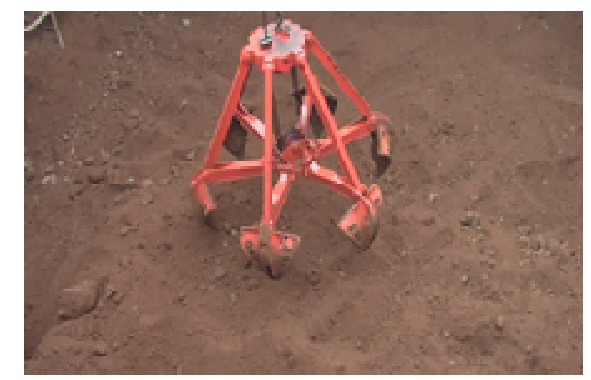

Fig. 3. 10t hollowed six-petaloid grab bucket

Analysis of the grab process. Select the data of the lifting motor and the open-close motor to generate a line chart. Based on the cyclical of the waveform from the line chart, we can get a complete grab operation graph. As is shown in Fig. 4, the blue waveform is for the lifting motor current and the 
red waveform for the open-close motor current. Ordinate stands for the voltage value, the thick horizontal line in the bottom is $0 \mathrm{~V}$, and each cell represents $1 \mathrm{~V}$; abscissa stands for time. And other current curves in this paper are the same with this.

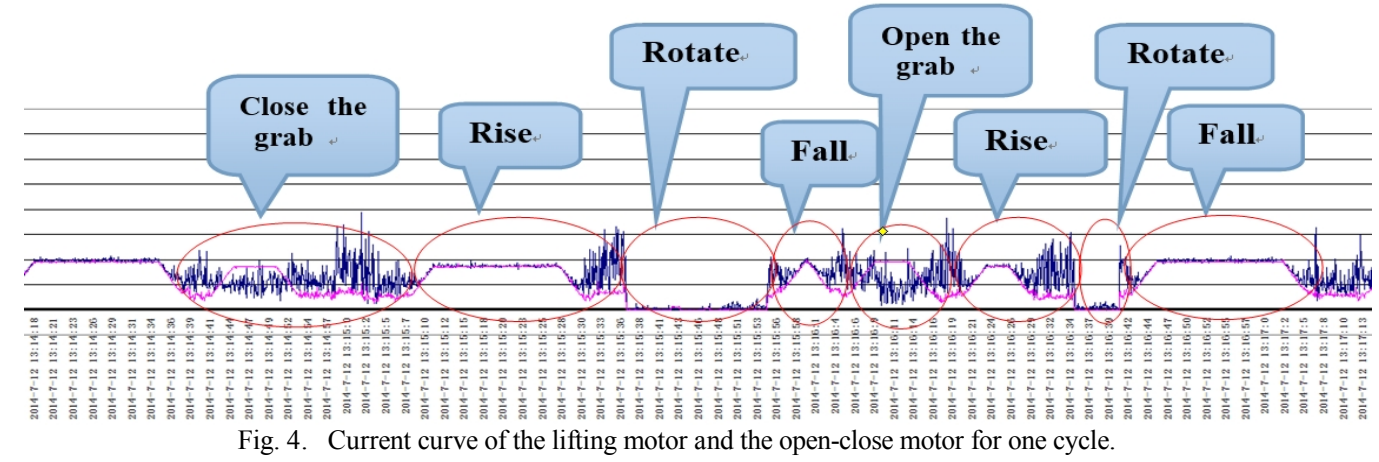

Analysis of the grab handling efficiency. Grab handling efficiency considers the quantity of grabbing material per unit time. It is usually expressed in tons per hour. The weight of the grabbing material is obtained from the torque limiter. After converting torque limiter data into a line chart, it can be easily found that the value of the torque limiter becomes peak and steady when the grab bucket on rise. This stable value is equal to the quantity of the grabbing material plus the weight of the bucket itself. And the time can be read directly from the data.

Table 1. Analysis of the Closing Time and Efficiency

\begin{tabular}{|l|c|c|}
\hline \multicolumn{1}{|c|}{ Type } & Traditional grab & Hollowed grab \\
\hline $\begin{array}{l}\text { Average closing time } \\
\text { (second) }\end{array}$ & $36.92 \mathrm{~s}$ & $27.52 \mathrm{~s}$ \\
\hline $\begin{array}{l}\text { standard deviation of the closing time } \\
\text { (second) }\end{array}$ & $8.29 \mathrm{~s}$ & $5.49 \mathrm{~s}$ \\
\hline Grab handling efficiency (tons per hour) & $82.6 \mathrm{t} / \mathrm{h}$ & $110 \mathrm{t} / \mathrm{h}$ \\
\hline
\end{tabular}

According to Table 1, we can draw the following conclusion. The grab handling efficiency is largely increased from 82.6 tons per hour using traditional grab bucket to $110 \mathrm{t} / \mathrm{h}$ using the new hollowed one. What is more, the new hollowed one takes 9 seconds less than the traditional grab bucket for the average close time.

Analysis of the grab handling stability. Overloading and other unstable situation are usually occurred during the closing period of grab bucket. So, for the stability analysis, the key variable is the current of lifting motor and open-close motor during that period.

Fig. 5 shows the working condition of the new hollowed grab bucket. There occurs some larger torque during the bucket close process. The current of open-close motor is stable and never go to zero.

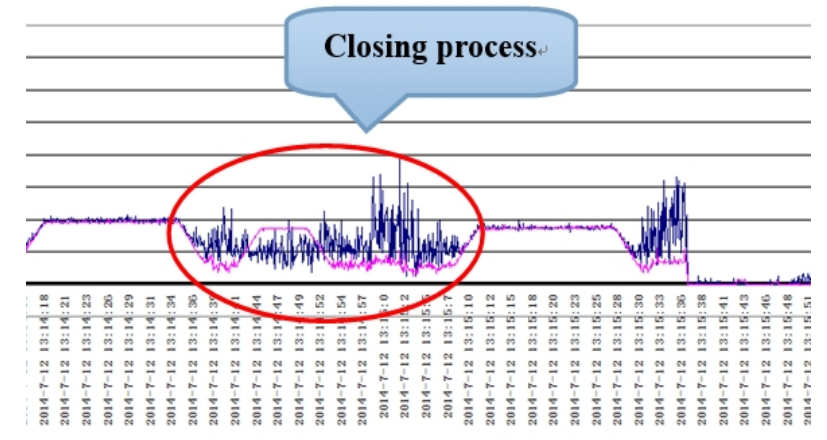

Fig. 5. Typical current curve of the hollowed six-petaloid grab bucket

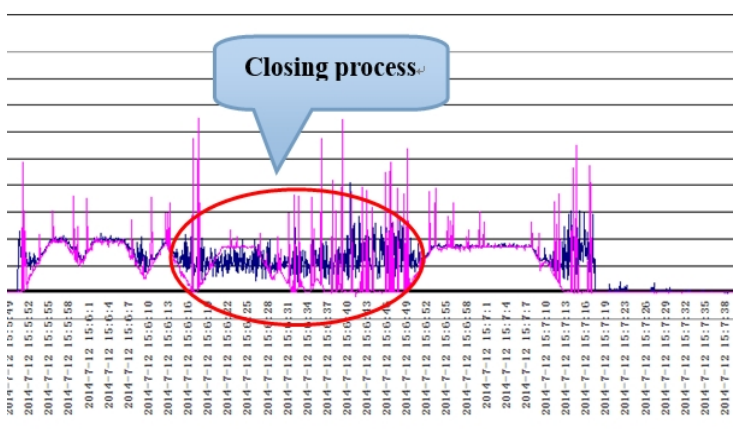

Fig. 6. Typical current curve of the traditional six-petaloid grab bucket

Fig. 6 shows the working condition of the traditional grab bucket. There also occurs some larger torque during the bucket close process. But the current of open-close motor is not stable. There is also zero ampere of the open-close motor current with multiple pulses. 
According to the test data, the close process can be divided into two phases regardless of the type of the grab bucket, which are Phase I and Phase II. As is shown in Fig. 7, Phase I refers to the early period of closing, there is a peak during that period. Phase II refers to the later period of closing, the current law is different.

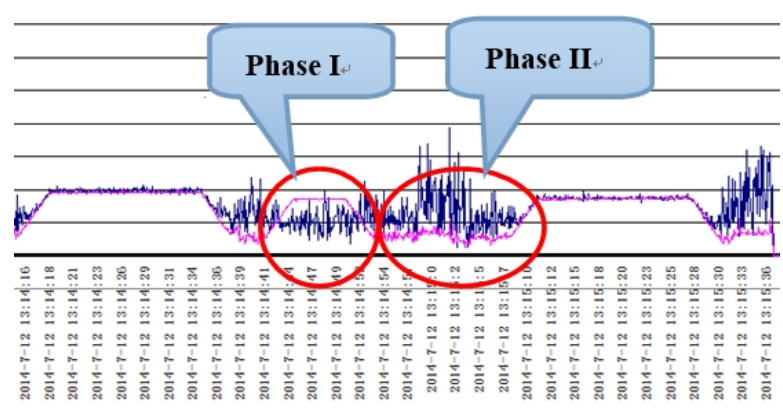

Fig. 7. Phase I and Phase II of the closing process

Based on the collected data, it would be more visually to compare the stability of the two grabs, the average current of the lifting and the open-close motor reflects the grab stress condition which is related to the property of grabbing material and the weight of the bucket. The value of the standard deviation and extra wave number reflects the fluctuation of motor current and stability of the system. Table 2 is the average value of these variables.

Table 2. Analysis of the Working Stability during the Closing Period

\begin{tabular}{|c|c|c|c|c|c|}
\hline \multirow[t]{3}{*}{ Type } & \multicolumn{5}{|c|}{ Closing process } \\
\hline & \multicolumn{2}{|c|}{ Lifting motor(Phase I , II) } & \multicolumn{3}{|c|}{ Open-close motor(phase II) } \\
\hline & $\begin{array}{l}\text { Average current } \\
\text { (Ampere) }\end{array}$ & $\begin{array}{l}\text { Standard deviation of the current } \\
\text { (Ampere) }\end{array}$ & $\begin{array}{l}\text { Extra } \\
\text { number } \\
\text { (times) }\end{array}$ & \begin{tabular}{|l|l|} 
wave & $\begin{array}{l}\text { Average current } \\
\text { (Ampere })\end{array}$ \\
\end{tabular} & $\begin{array}{l}\text { Standard deviation of the } \\
\text { current } \\
\text { (Ampere) }\end{array}$ \\
\hline hollowed grab & $1.2517 \mathrm{~A}$ & $0.4466 \mathrm{~A}$ & 1.7419 & $0.7951 \mathrm{~A}$ & $0.4940 \mathrm{~A}$ \\
\hline traditional grab & $1.3430 \mathrm{~A}$ & $0.4752 \mathrm{~A}$ & 2.2000 & $0.8152 \mathrm{~A}$ & $0.6560 \mathrm{~A}$ \\
\hline
\end{tabular}

Obviously, the working stability of the new hollowed grab bucket is much better than the traditional one for the standard deviation is 0.494 to 0.656 . What is more, in phase II, the extra wave number is decreased from 2.2 using traditional grab bucket to 1.24 using the new hollowed one.

\section{Conclusion}

Practice proves that this grab operation test system is successful. It is quite efficient to analyze the grab operation rules, operational efficiency and stability through this system, providing a solid theoretical basis for the further improvement of the grab bucket and upgrading of the intelligent control method.

\section{References}

[1] Jintao Hu, "Research of Key Technologies for Intelligent grab ship," Wuhan university of technology, May 2013;

[2] Bin Sun, WANG Xu-chang, Performance optimization of automatic ship unloading for GSU, Journal of Mechanical \& Electrical Engineering Vol . 28 No . 6 Jun . 2011.

[3] Jianfeng Yang, "General grab operation for nickel ore” Port Operation 2013. No. 5(Serial No. 212). 